\title{
Tuberculosis screening in patients receiving biological therapy
}

\author{
Ramos $\mathrm{S}^{1}$, Nogueira $\mathrm{A}^{2}$, Dias A ${ }^{1}$, Gonçalves $\mathrm{AF}^{3}$, Gaio AR ${ }^{4}$, Duarte R 5
}

\section{ABSTRACT}

Aim: Biological therapies are a risk factor for tuberculosis (TB). Portuguese recommendations endorse universal baseline screening for TB, before starting biologics (2006) and annually thereafter if screened negative (2012 update). The gain with re-screening remains unknown. We aimed to: i) identify the risk of latent TB infection at baseline screening among patients candidates to initiate biologics; ii) present follow-up results for patients receiving different biological therapies and analyse intolerance or toxicity related to preventive therapy, conversions of immunodiagnostic tests under biological therapy and development of active TB.

Methods: Patients screened for TB at a reference centre before starting biological therapy between 2008-2012 were identified. Medical files were retrospectively reviewed. Demographic data, screening and follow-up results and information on biological therapy were collected.

Results: 183 patients were included in the study, with 115 starting biological therapy. The baseline screening was positive in $52(45.2 \%)$ patients - 50(96.2\%) were proposed for preventive treatment ( 2 had abnormal liver enzymes). Mild hepatotoxicity occurred in 4(8\%) patients without need to interrupt TB prophylaxis. No

1. Department of Clinical Epidemiology, Predictive Medicine and Public Health, Faculty of Medicine, University of Porto, Portugal 2. Department of Mathematics, Faculty of Science, University of Porto, Portugal

3. Pulmonology Department, Centro Hospitalar de Vila Nova de Gaia/Espinho EPE, Portugal

4. Department of Mathematics, Faculty of Science, University of Porto, Portugal; Centre of Mathematics, University of Porto,

Portugal

5. Department of Clinical Epidemiology, Predictive Medicine and Public Health, Faculty of Medicine, University of Porto, Portugal; EPIUnit Institute of Public Health, University of Porto, Portugal; Chest Disease Centre Vila Nova de Gaia, Portugal; Pulmonology Department, Centro Hospitalar de Vila Nova de Gaia/Espinho EPE, Portugal cases of active TB occurred during follow-up in patients with positive baseline screening. Among the 63(54.8\%) patients who screened negative, 2(3.2\%) developed active TB (under infliximab and adalimumab) more than one year after initiation of biologics. 26(41.3\%) patients were re-screened at the TB centre. 5(19.2\%) had tuberculin skin test (TST) conversion and one concomitantly undetermined IGRA. No IGRA conversions were observed. The follow-up period was 4.0 years. TB baseline screening's negative predictive value (NPV) was 96.8\% (95\%CI: $89.0 \%$ to $99.5 \%$ ). A low rate of rescreening was observed.

Conclusion: The rate of latent TB at baseline screening was higher than expected. Preventive treatment was well tolerated. No patients with positive baseline screening developed active TB. Efforts should be made to raise awareness concerning the risk of $\mathrm{TB}$ exposure, specially considering that the active TB cases were compatible with new infection. The rate of re-screening suggests a low awareness regarding current recomendation. Nation-wide studies are necessary to evaluate the efficacy of the re-screening strategy and to clarify what risk groups most benefit from it.

Keywords: Screening; Latent tuberculosis; Biological agents; Anti-tumor necrosis factor-alpha therapy; Tuberculosis

\section{INTRODUCTION}

The advent of biological therapies has greatly improved the treatment and management of immunomediated diseases such as Crohn's disease, psoriasis, rheumatoid arthritis, ankylosing spondylitis and multiple sclero$\mathrm{sis}^{1-3}$, allowing for a better quality of life for these patients ${ }^{4}$. Biologic drugs are now a staple in the clinical approach to severe forms of these diseases. However, they require careful management given their suppres- 
sive effect on the patient's immune system ${ }^{5}$. Therefore, they pose as a risk factor for the development of active tuberculosis $(\mathrm{TB})^{6,7}$.

Medical societies have introduced recommendations for screening of latent tuberculosis infection before initiation of biological therapy ${ }^{6,8}$. Portugal is a country with an intermediate incidence of TB (25.2/100 000 habitants, data of 2012) ${ }^{9}$. National recommendations published in $2006^{10}$ consisted of a TB screening prior to the initiation of biological therapy as well as an annual screening afterwards for those with a negative baseline screening, for as long, as the therapy is maintained (updated in 2012) ) $^{11,12}$. Patients with a positive baseline screening must be regularly questioned by their assistant physicians in order to identify recent TB exposure with the shortest delay possible ${ }^{12}$.

The effectiveness of the baseline screening has been confirmed $^{13,14}$, but to date the gain in the annual re-screening strategy remains unknown.

The present study was conducted at an urban reference centre for TB. This TB centre is responsible for the management of all outpatients TB cases in the region and the screening of risk populations. An existing regional network crosses information of active TB cases diagnosed by clinical or laboratorial means as well as by epidemiologic inquiries ensuring notification of all diagnosed cases. Patients who are candidates for initiation of biological therapy are sent to the reference centre by their assistant physician in order to be screened for TB before the beginning of the therapy. Contact between the assistant physician and the reference centre doctor is promoted in the daily clinical practice.

The aim of this study was to identify the risk of latent tuberculosis infection (LTBI) at baseline screening among patients candidates to initiate biological therapies and to present the follow-up results for patients receiving different biological therapies (either with positive or negative screening). We analysed intolerance or toxicity related to preventive therapy, conversions of immunodiagnostic tests for those with a negative baseline screening and development of active TB for all.

\section{METHODS}

Clinical files of all patients that have been screened for $\mathrm{TB}$ at the reference TB centre as candidates to start biological therapy, between January 2008 and December
2012, were retrospectively reviewed. All patients were followed in order to evaluate development of active $\mathrm{TB}$, intolerance or toxicity related to preventive therapy and seroconversions among those with a negative screening (annual re-screening). The follow-up of the study was concluded in 2014.

Exclusion criteria was unavailability of information regarding initiation of biologic therapy.

After exclusion of active TB, screening methodology includes a detailed medical history, physical examination, chest radiography, tuberculin skin test (TST) and interferon- $\gamma$ release assay (IGRA). A positive TST was considered when the diameter of the transverse induration surpassed 10mm in immunocompetent patients or $5 \mathrm{~mm}$ in immunosuppressed patients. Quantiferon-TB Gold in-tube (Cellestis Ltd, Victoria, Australia) was used to determine the QTF assay according to manufacturer's instructions. IGRA was considered to be positive if IFN- $\gamma \geq 0.35 \mathrm{IU} / \mathrm{ml}$. All patients with past non-treated TB, recent TB exposure or a positive TST or IGRA were offered preventive therapy, after exclusion of risk factors for toxicity.

Patients were considered immunosuppressed when receiving immunosuppressive therapy, such as corticosteroids in doses $>15 \mathrm{mg}$ /day for more than 2 weeks.

The demographic and clinical data of the patients, baseline screening, follow-up results and information regarding the biological therapy were collected from clinical records.

\section{STATISTICAL ANALYSIS}

A descriptive analysis of outcomes and prevalence study of tuberculosis was performed in the patients who started biological therapy. Continuous variables are expressed as mean \pm standard deviation (SD), while nominal variables are presented in absolute and percentage values (\%). Statistical analysis was conducted using SPSS Statistics V.22.0 for Windows software package.

\section{RESULTS}

During the studied period, 183 patients were included (38.8\% with dermatological, 29.5\% with gastroenterological, $16.9 \%$ with rheumatological, and $14.7 \%$ with neurological diseases). From these, 115 (62.8\%) patients did start biological therapy. The mean 
\pm standard deviation of the age of the 115 patients who initiated biological therapy was $41 \pm 11.5$ years-old ( $\min =18$ years-old, $\max =67$ years-old). The mean follow-up period of this study was 4.0 years.

The baseline screening of the 115 patients who started biological therapy proved to be negative in 63 (54.8\%) patients and positive in 52 (45.2\%) (Table I). All 52 patients with positive screening were considered for preventive treatment -2 patients did not receive treatment due to hepatic co-morbidity. The regimens used for preventive therapy included 9 months of isoniazid (92\%), 3 months isoniazid-rifampicin (4\%), 4 months of rifampicin (2\%) and 12 months isoniazid (2\%). Toxicity was reported in $5(10 \%)$ cases. Hepatotoxicity was found in 4 cases but all completed their treatment successfully. One patient had dermatologic toxicity and was unable to complete his treatment. In all cases the situation was rapidly resolved and there was no need for hospitalization. One patient abandoned preventive treatment after 2 months of isoniazid without reported adverse events.

Out of the 63 patients with negative baseline screening, $26(41.3 \%)$ patients were re-screened on follow-up at the reference centre. 5 (19.2\%) cases of TST conversion occurred during follow-up and 1 (3.9\%) case had concomitant undetermined IGRA. Regarding the cases of TST conversion, 3 patients were female, mean age of 29.2 ( $\min =22, \max =32)$. None had previous TB history nor past exposure. 2 patients had multiple sclerosis, other 2 had psoriasis and 1 had Crohn's disease. They were treated with natalizumab (2), infliximab (2) and etanercept (1). New TB exposure since the baseline screening was not reported. No IGRA conversions were observed. The follow-up screening results are summarized in Table II.

No cases of active TB were observed in the patients with positive baseline screening until conclusion of follow-up. There were 2 (3.2\%) reported cases of active $\mathrm{TB}$ among the 63 patients with negative baseline screening. One was male and the other female. The younger was 37 years old and the older 61 years old. One had Crohn's disease and was treated with infliximab, whilst the other had rheumatoid arthritis and received adalimumab. Neither one had previous TB history nor past exposure, but one patient reported new TB exposure during treatment with the biologic agent. In both cases we observed extra-pulmonary $\mathrm{TB}$, namely pleural and soft tissues TB. One case of active TB developed after 1 year, while the other 2 years after the beginning of the treatment. Neither of these two patients

\section{TABLE I. CHARACTERISTICS OF THE PATIENTS WHO STARTED BIOLOGICAL THERAPY}

\begin{tabular}{|c|c|}
\hline & $\mathbf{N}$ \\
\hline \multicolumn{2}{|l|}{ Gender } \\
\hline Total & 115 \\
\hline Male & $60(52.2)$ \\
\hline Female & $55(47.8)$ \\
\hline \multicolumn{2}{|l|}{ Underlying disease } \\
\hline Total & 115 \\
\hline Psoriasis & $45(39.1)$ \\
\hline Crohn's disease & $21(18.3)$ \\
\hline Multiple sclerosis & $21(18.3)$ \\
\hline Ankylosing spondylitis & $12(10.4)$ \\
\hline Rheumatoid arthritis & $8(7)$ \\
\hline Other & $8(7)$ \\
\hline \multicolumn{2}{|l|}{ Immunosuppression } \\
\hline Total & 115 \\
\hline Yes & $62(53.9)$ \\
\hline No & $52(45.2)$ \\
\hline Missing & $1(0.9)$ \\
\hline \multicolumn{2}{|l|}{ TB exposure } \\
\hline Total & 115 \\
\hline Yes & $20(17.4)$ \\
\hline No & $95(82.6)$ \\
\hline \multicolumn{2}{|l|}{ TB history } \\
\hline Total & 115 \\
\hline None & $107(93.0)$ \\
\hline Previous TB treatment & $7(6.1)$ \\
\hline Sequela on the x-ray & $1(0.9)$ \\
\hline \multicolumn{2}{|l|}{ Biological therapy } \\
\hline Total & 115 \\
\hline Anti-TNF $\alpha$ & $75(65.2)$ \\
\hline Etanercept & $25(33.3)$ \\
\hline Adalimumab & $24(32.0)$ \\
\hline Infliximab & $20(26.7)$ \\
\hline Golimumab & $6(8.0)$ \\
\hline Anti-CD20 & $3(2.6)$ \\
\hline Ocrelizumab & $2(66.7)$ \\
\hline Rituximab & $1(33.3)$ \\
\hline Other classes & $37(32.2)$ \\
\hline Natalizumab & $24(64.9)$ \\
\hline Ustekinumab & $10(27.0)$ \\
\hline Tocilizumab & $2(5.4)$ \\
\hline Efalizumab & $1(2.7)$ \\
\hline
\end{tabular}

Other diseases - hidradenitis suppurativa, necrobiosis lipoidica, spondiloarthropathy, psoriatic arthritis, non-specified inflammatory bowel disease 
TABLE II. FOLLOU-UP SCREENING RESULTS

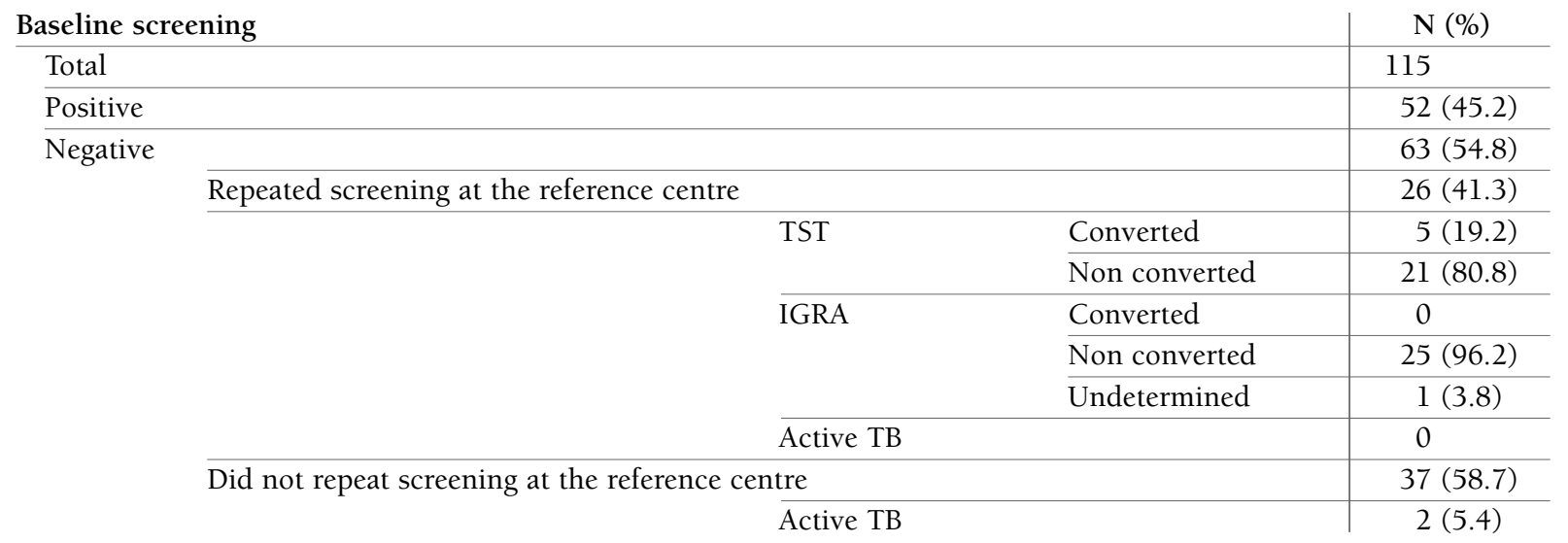

had repeated screening after initiation of biologic therapy.

The negative predictive value (NPV) of the TB baseline screening was $96.8 \%$ (95\% CI:89.0\% to $99.5 \%$ ).

\section{DISCUSSION}

In the group of patients who started biologic agents, $45 \%$ were found to have latent TB at the baseline screening. The preventive treatment was mostly well tolerated with few adverse effects. Most of the patients undergoing biologics did not repeat screening. A high rate of TST conversion was observed. Two cases of active TB were diagnosed in patients with negative baseline screening.

The suppressive effect of biological drugs is a significant concern, as drugs such as anti-TNF have been shown to interfere with granuloma formation. The granuloma is a central part of the defence mechanism against Mycobacterium tuberculosis and the maintenance of its structure is highly dependent of TNF. Therefore, anti-TNF drugs decrease the host ability to defend against the pathologic agent and the risk of developing active TB rises 5 .

The rate of latent $\mathrm{TB}$ at the baseline screening was higher than expected (45.2\%), when compared with background population $(15.15 \%)^{15}$. It is well known that a few underlying diseases may influence TB rates. Studies on patients with immunomediated diseases reported a prevalence of LTBI ranging from $6 \%$ to $30 \%{ }^{3,4,16}$. Such a result highlights the importance and supports the need for compliance with the recommendations regarding baseline screening. The baseline screening comprises both TST and IGRA as none has been yet proven to be clearly better for patients with immune-mediated inflammatory disease ${ }^{17}$.

Isoniazid is the primary preventive scheme for LTBI treatment. Given the severity of its potential adverse effects such as life threatening hepatotoxicity ${ }^{18}$, it is often viewed with concern by physicians. As reported in other studies ${ }^{19,20}$, we observed that preventive treatment was mostly well tolerated with few adverse effects with only 4 patients presenting mild hepatotoxicity, which did not prevent them from completing the treatment. On the other hand, one patient developed dermatologic toxicity. He was proposed for alternative preventive schemes with rifampicin but the attempts to avoid toxicity were fruitless and the treatment was suspended. There is the possibility that concomitant treatment with isoniazid and biologics may increase the risk of hepatotoxicity ${ }^{21,22}$ but our patients who started biologic drugs while still receiving preventive treatment did not report pharmacologic interactions, which supports the belief that physicians may safely introduce biologic therapy during preventive treatment.

We observed a very good compliance with preventive treatment with only two patients not completing it. One was due to dermatologic toxicity; the other abandoned the treatment without reported adverse events, after two months of isoniazid.

Despite appropriate baseline screening, some patients may develop TB during biological therapy ${ }^{23,24}$. Recommendations issued in 2012 advocate annual TB 
screening to patients undergoing biological therapy who had a negative baseline screening ${ }^{12}$. In our study, only $41.3 \%$ of our patients with negative baseline screening repeated the screening during follow-up at the reference centre. However, we cannot fully evaluate compliance with the recommendations considering that the update regarding annual screening was published in 2012 and our study includes patients with different treatment durations and/or who suspended therapy before the publication of the update.

During follow-up, we observed 5 (19.2\%) TST conversions, though only a single concomitant undetermined IGRA. Similarly to findings of several studies, the correspondence between results of TST and IGRA is rather poor ${ }^{25-28}$. IGRA is considered the best screening tool for latent TB infection in patients receiving biologic drugs, as TST carries a heavier set of confounding factors, such as the booster effect and a lower specificity ${ }^{6,24,29}$. In addition, the patient's underlying disease may independently interfere with TST results. For instance, rheumatoid arthritis patients naive to biological therapy present a reduced recall response which may lead to false negative TST results ${ }^{5,26}$ and psoriasis patients also display a lower reactivity to $\mathrm{TST}^{4}$. However, it is still debatable whether the IGRA results are more accurate than TST for the TB screening of patients undergoing anti-TNF $\alpha$, as findings vary considerably between studies ${ }^{16,25,26}$. As IGRA relies on the production of IFN- $\gamma$, which is known to be decreased by immunossupressive therapies, the effectiveness of IGRA in detecting LTBI may consequently be reduced ${ }^{25,30}$. We cannot, therefore, confidently consider these conversions as latent TB infection, but cannot exclude the possibility either. Nevertheless, while consensus on the best screening method is not achieved, patients with TST conversion at re-screening represent a risk that should not be dismissed.

While there were no cases of active TB in patients with positive baseline screening, 2 cases were reported in patients with negative baseline screening. One patient had rheumatoid arthritis while the other was diagnosed with Crohn's Disease. Both developed the disease more than one year after the beginning of biological therapy, which suggests new infection, rather than re-activation. Actually, one of the patients had exposure to active $\mathrm{TB}$ while receiving the biologic agent though it was only discovered at the time of diagnosis. The other patient did not report exposure to TB. The patients were being treated with infliximab and adalimumab. It is well known that TB development occurs more frequently with infliximab ${ }^{31,32}$. Moreover, infliximab and adalimumab are associated with the earliest onset of the disease ${ }^{4}$. Our findings are consistent with the literature, indicating that antibodies infliximab and adalimumab possess a greater risk of TB progression, when compared with soluble TNF receptor such as etanercept ${ }^{2,4,32,33}$. This might suggest that patients under these specific biological drugs require a higher degree of vigilance during follow-up.

We obtained a NPV of $96.8 \%$ for the baseline TB screening as defined in the national recommendations. Publications in the literature have achieved similar results $^{34}$. It is compatible with our findings, as our cases of active TB were diagnosed more than one year after initiation of biological therapy. We may therefore infer that current baseline screening recommendations are appropriate for identifying LTBI.

It has been suggested that prescribers of biologics are not sufficiently aware of the annual screening recommendation $s^{35}$. Therefore, bringing the recommendations to attention of prescribers, as well as the development of a joined effort between reference centres and prescribers may increase the implementation of the annual TB screenings.

The present study carries certain limitations, the first being its small size. The number of patients included in the study is not fully representative of the actual number of patients who have received biological therapy during the studied period of time, as a result of a very high proportion of unknown data regarding the start of the biological drugs. The study also lacked a control group of patients for result comparison. In addition, very few patients repeated screening at the reference centre, which accounts for a small number of conversions resulting in low statistical power. We were therefore unable to find common variables among these patients, which might have helped in further identifying high-risk individuals who would most benefit from annual re-screening. Also, our study was performed in a country with intermediate TB incidence, so our results may not apply to countries with low TB incidence. Last but not the least, the greatest limitation of this and most studies on latent TB screening lies in the absence of a gold standard for the diagnosis of latent TB.

Despite these limitations, the study has several strong assets. First of all, it was conducted at a TB reference centre that crosses information both from inpatients and outpatients. It also includes information from several clinical specialties that deal with biolo- 
gics, allowing for a more global understanding of the present situation. Additionally, all cases of diagnosed active TB are notified so we can ensure that no other patients have developed the disease in the studied population.

\section{CONCLUSION}

The baseline TB screening has showed a higher than expected rate of latent tuberculosis in the candidates to start biological therapy. Adverse events were not frequent and most did not interfere with the completion of the preventive treatment. A high incidence of TST conversions and cases of active TB has been observed, alerting to the necessity of careful follow-up of the patients undergoing biological therapy. Moreover, both cases of active TB suggest new infection, highlighting the importance of the physician's role in preventing such outcomes. The compliance with the recommendations regarding annual screening could not be fully evaluated though it appears to not yet be implemented in daily practice. Accordingly, we believe efforts should be made to raise awareness of prescribing physicians concerning the risk of exposure to TB. Meanwhile, larger nation-wide studies should be designed in order to evaluate the efficacy of the re-screening strategy and to clarify which risk groups most benefit with annual re-screening.

\section{CORRESPONDENCE TO}

\section{Raquel Duarte}

Department of Clinical Epidemiology,

Predictive Medicine and Public Health,

Faculty of Medicine, University of Porto, Portugal

E-mail: rdmelo@med.up.pt

\section{REFERENCES}

1. He D, Bai F, Zhang S et al. High incidence of tuberculosis infection in rheumatic diseases and impact for chemoprophylactic prevention of tuberculosis activation during biologics therapy. Clin Vaccine Immunol 2013; 20:842-847.

2. Abreu C, Magro F, Santos-Antunes J et al. Tuberculosis in anti-TNF-alpha treated patients remains a problem in countries with an intermediate incidence: analysis of 25 patients matched with a control population. J Crohns Colitis 2013; 7:e486-492.

3. Sauzullo I, Mengoni F, Marocco R et al. Interferon-gamma release assay for tuberculosis in patients with psoriasis treated with tumour necrosis factor antagonists: in vivo and in vitro analysis. Br J Dermatol 2013; 169:1133-1140.

4. Sanchez-Moya AI, Garcia-Doval I, Carretero G et al. Latent tuberculosis infection and active tuberculosis in patients with psoriasis: a study on the incidence of tuberculosis and the prevalence of latent tuberculosis disease in patients with moderate- severe psoriasis in Spain. BIOBADADERM registry. J Eur Acad Dermatol Venereol 2013; 27:1366-1374.

5. Solovic I, Sester M, Gomez-Reino JJ et al. The risk of tuberculosis related to tumour necrosis factor antagonist therapies: a TBNET consensus statement. Eur Respir J 2010; 36:1185-1206.

6. Fuchs I, Avnon L, Freud T and Abu-Shakra M. Repeated tuberculin skin testing following therapy with TNF-alpha inhibitors. Clin Rheumatol 2009; 28:167-172.

7. Garcovich S, Ruggeri A, D’Agostino M et al. Clinical applicability of Quantiferon-TB-Gold testing in psoriasis patients during long-term anti-TNF-alpha treatment: a prospective, observational study. J Eur Acad Dermatol Venereol 2012; 26:1572-1576 .

8. Hatemi G, Melikoglu M, Ozbakir F, Tascilar K and Yazici H. Quantiferon-TB Gold in tube assay for the screening of tuberculosis before and during treatment with tumor necrosis factor alpha antagonists. Arthritis Res Ther 2012; 14:R147.

9. Tuberculosis surveillance and monitoring in Europe, in European Centre for Disease Prevention and Control/ WHO Regional. Office for Europe. 2013: Stockholm: European Centre for Disease Prevention and Control, 2013.

10. Fonseca JE, Lucas H, Canhao $\mathrm{H}$ et al. [Guidelines for the diagnosis and treatment of latent tuberculosis infection and active tuberculosis in patients with inflammatory joint diseases proposed for treatment with tumour necrosis factor alpha antagonist drugs]. Rev Port Pneumol 2006; 12:603-613.

11. Fonseca JE, Lucas H, Canhao H et al. Recommendations for the diagnosis and treatment of latent and active tuberculosis in inflammatory joint diseases candidates for therapy with tumor necrosis factor alpha inhibitors: March 2008 update. Acta Reumatol Port 2008; 33:77-85.

12. Duarte R, Campainha S, Cotter J et al. Position paper on tuberculosis screening in patients with immune mediated inflammatory diseases candidates for biological therapy. Acta Reumatol Port 2012; 37:253-259.

13. Carmona L, Gomez-Reino JJ, Rodriguez-Valverde V et al. Effectiveness of recommendations to prevent reactivation of latent tuberculosis infection in patients treated with tumor necrosis factor antagonists. Arthritis Rheum 2005; 52:1766-1772.

14. Papay P, Primas C, Eser A et al. Retesting for latent tuberculosis in patients with inflammatory bowel disease treated with TNF-alpha inhibitors. Aliment Pharmacol Ther 2012; 36:858-865.

15. Lopes JS, Rodrigues P, Pinho ST, Andrade RF, Duarte R and Gomes MG. Interpreting measures of tuberculosis transmission: a case study on the Portuguese population. BMC Infect Dis 2014; $14: 340$

16. Jauregui-Amezaga A, Turon F, Ordas I et al. Risk of developing tuberculosis under anti-TNF treatment despite latent infection screening. J Crohns Colitis 2013; 7:208-212.

17. Pai M, Denkinger CM, Kik SV et al. Gamma interferon release assays for detection of Mycobacterium tuberculosis infection. Clin Microbiol Rev 2014; 27:3-20.

18. Lobue P and Menzies D. Treatment of latent tuberculosis infection: An update. Respirology 2010; 15:603-622.

19. Bourre-Tessier J, Arino-Torregrosa M and Choquette D. Increased incidence of liver enzymes abnormalities in patients treated with isoniazid in combination with disease modifying and/or biologic agents. Clin Rheumatol 2014; 33:1049-1053.

20. Hsia EC, Cush JJ, Matteson EL et al. Comprehensive tuberculosis screening program in patients with inflammatory arthritides treated with golimumab, a human anti-tumor necrosis fac- 
tor antibody, in Phase III clinical trials. Arthritis Care Res (Hoboken) 2013; 65:309-313.

21. Ghabril M, Bonkovsky HL, Kum C et al. Liver injury from tumor necrosis factor-alpha antagonists: analysis of thirty-four cases. Clin Gastroenterol Hepatol 2013; 11:558-564.e553.

22. Parekh R and Kaur N. Liver Injury Secondary to Anti-TNF-Alpha Therapy in Inflammatory Bowel Disease: A Case Series and Review of the Literature. 2014; 2014:956463.

23. Hatzara C, Hadziyannis E, Kandili A et al. Frequent conversion of tuberculosis screening tests during anti-tumour necrosis factor therapy in patients with rheumatic diseases. Ann Rheum Dis 2014.

24. Debeuckelaere C, De Munter P, Van Bleyenbergh P et al. Tuberculosis infection following anti-TNF therapy in inflammatory bowel disease, despite negative screening. J Crohns Colitis 2014; 8:550-557.

25. Drago L, Nicola L, Signori V et al. Dynamic QuantiFERON Response in Psoriasis Patients Taking Long-Term Biologic Therapy. Dermatol Ther (Heidelb) 2013; 3:73-81.

26. Goletti D, Sanduzzi A and Delogu G. Performance of the tuberculin skin test and interferon-gamma release assays: an update on the accuracy, cutoff stratification, and new potential immune-based approaches. J Rheumatol Suppl 2014; 91:24-31.

27. Sanchez-Moya AI and Dauden E. Incidence of tuberculosis infection in psoriatic patients on anti-TNF therapy: report of a case series with 144 patients. J Eur Acad Dermatol Venereol 2011; 25:730-733.

28. Andrisani G, Armuzzi A, Papa A et al. Comparison of Quantiferon-TB Gold versus tuberculin skin test for tuberculosis screening in inflammatory bowel disease patients. J Gastrointestin Liver Dis 2013; 22:21-25.
29. Dominguez J, Vilavella M and Latorre I. Interferon gamma assays in the diagnosis of tuberculosis infection in psoriasis patients who are candidates for biologic therapies. Actas Dermosifiliogr 2012; 103:880-886.

30. Hamdi H, Mariette X, Godot V et al. Inhibition of anti-tuberculosis T-lymphocyte function with tumour necrosis factor antagonists. Arthritis Res Ther 2006; 8:R114.

31. Ribeiro C, Correia AM and Duarte R. Tuberculosis and TNFalpha antagonists— what are we missing? Rev Port Pneumol 2014; 20:53-54.

32. Nacci F and Matucci-Cerinic M. Tuberculosis and other infections in the anti-tumour necrosis factor-alpha (anti-TNF-alpha) era. Best Pract Res Clin Rheumatol 2011; 25:375-388.

33. Tubach F, Salmon D, Ravaud P et al. Risk of tuberculosis is higher with anti-tumor necrosis factor monoclonal antibody therapy than with soluble tumor necrosis factor receptor therapy: The three-year prospective French Research Axed on Tolerance of Biotherapies registry. Arthritis Rheum 2009; 60:1884-1894 .

34. Campainha S, Gomes T, Carvalho A and Duarte R. Negative predictive value of TST and IGRA in anti-TNF treated patients. Eur Respir J 2012; 40:790-791

35. Xerinda S, Oliveira O, Lucas R, Fonseca JE, Varela P, Cotter J, Duarte R.Barriers for tuberculosis screening in patients starting biologic drugs in Portugal. 2014, Medical School, University of Porto: Manuscript submitted for publication. 